\title{
RF SYSTEMS FOR THE BETATRON-NODE SCHEME EXPERIMENT AT LBNL*
}

\author{
S. Lidia $\dagger$, S. De Santis, LBNL, Berkeley, CA USA \\ T. Houck, LLNL, Livermore, CA USA
}

\begin{abstract}
Two-beam accelerators based upon relativistic klystron's (RK's) have been proposed as power sources for future generation linear electron-positron colliders. These drivers are known to suffer from several transverse beam break-up (BBU) instabilities. A program to study a particular technique (the 'betatron node scheme') for ameliorating the high frequency BBU is under way at LBNL. Central to this study are the pillbox RF cavitities and RF beam position monitors (BPM's) employed. This paper describes the design, fabrication, and testing of the RF components. Performance details during operation are also discussed.
\end{abstract}

\section{INTRODUCTION}

The Betatron Node Scheme (BNS) experiment at LBNL has now demonstrated reduction of cumulative BBU amplitude growth by more than 13 e-folds over a 10period beamline. The successful demonstration of the technique used to damp the BBU growth relies upon the careful design of RF cavities and beam position monitors used therein. The outline of the paper is as follows. Section 2 describes the layout of the BNS experiment. Details of the RF component designs are given in Section 3. Measured values of the cavity parameters and the BPM response functions are given in Section 4. Two different modes of operation and data acquisition are described in Section 5, along with a discussion of the current results. Observations and conclusions are then drawn in Section 6.

\section{DESCRIPTION OF BNS EXPERIMENT}

The BNS experiment is described in greater detail elsewhere [1]. The experiment measures growth in the high-frequency transverse oscillation of a $250 \mathrm{~ns}$ (FWHM), $500 \mathrm{~A}, 1 \mathrm{MeV}$ electron beam. This cumulative BBU mode is common in all linear accelerators and beamlines with trapped dipole modes in periodically spaced RF structures. The beamline is composed of a set of 10 periods. A period is made up of one RF pillbox cavity, one capacitive button beam position monitor (BPM), one vacuum pumping port, three nipple flanges for vacuum containment, and three fine wire solenoids for beam focusing and transport. A schematic of a two-period length is shown in Figure 1.

\footnotetext{
*This work was supported by U.S. Department of Energy, Office of Science, Division of High Energy Physics Contracts DE-AC0376SF00098 (LBNL) and W-7405-ENG-48 (LLNL)
}

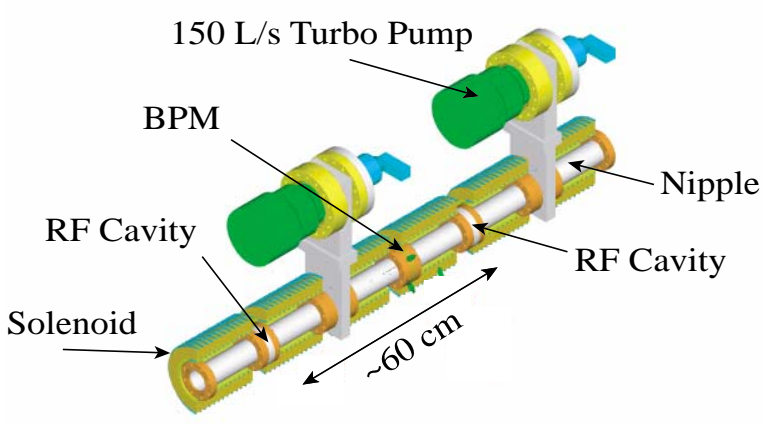

Figure 1: Schematic of BNS beamline.

\section{RF COMPONENT DESCRIPTIONS}

The choice of the dipole mode frequency excited within the cavities and detected at the BPMs was made by balancing the requirements of high frequency with high beam transmission. The high-frequency requirement ensures that the RF period is much less than the beam pulse rise time or flat top, and that steady-state conditions are achievable, To transmit the majority of the beam pulse through the system using reasonable solenoid fields was a first priority. Off-the-shelf components for the beamline were chosen whenever possible to help decrease the overall cost of the experiment. A relatively short betatron wavelength was required so that multiple betatron oscillations could be obtained with a fairly short $(<10 \mathrm{~m})$ long transport line.

We chose standard MDC 2" OD nipples with 3-3/8" conflat flanges as the basic building blocks of the system. This allows for a 1.87" ID aperture for beam transport. Each 6.45" long nipple supports a fine wire solenoid with $87 \mathrm{x} 14$ turns. $1-\mathrm{kW}$ or $3-\mathrm{kW}$ power supplies provide up to $16 \mathrm{~A}$ of current to either individual solenoids or solenoid triplets. Fields of up to $1.5 \mathrm{kG}$ can be easily produced. To minimize magnetic field holes, pumping ports were constructed from MDC GV-2000M gate valve bodies, equipped with Multi-Lam finger stock tack-welded onto the ID to provide a current-return path. Three nipples are used to form the periodic unit-cell of the beamline. Between each nipple is an MDC double-sided, 3-3/8" OD conflat flange that holds an RF cavity, a BPM, or a pumping port.

\subsection{Dipole Pillbox Cavities}

Physics, construction and assembly requirements dictated that single flange widths only be used between nipples, and that the solenoids are able to slide over the 
RF cavities. The OD of the pillbox cavity volume is limited to the 2" ID of the conflat flange. This dictated the use of dielectrics to load the cavities and lower the dipole mode frequencies so that the modes would be trapped. Additional stainless steel pipe sections were brazed and tack-welded onto the flange to hold the dielectric ring insert, as well as to provide an additional cutoff region for the mode.

We chose to use $96 \%$ Alumina as the dielectric material. The cavity gap itself is $2 \mathrm{~mm}$ wide, with a $26 \mathrm{~mm}$ outer radius, and a $16 \mathrm{~mm}$ inner radius defined by the cutoff pipe ID. The Alumina insert has a $26 \mathrm{~mm}$ outer radius and a $8 \mathrm{~mm}$ radial thickness. This produces a dipole $\mathrm{TM}_{110}$ mode with frequency $\sim 3.75 \mathrm{GHz}$. A schematic of the RF cavity is shown in Figure 2.

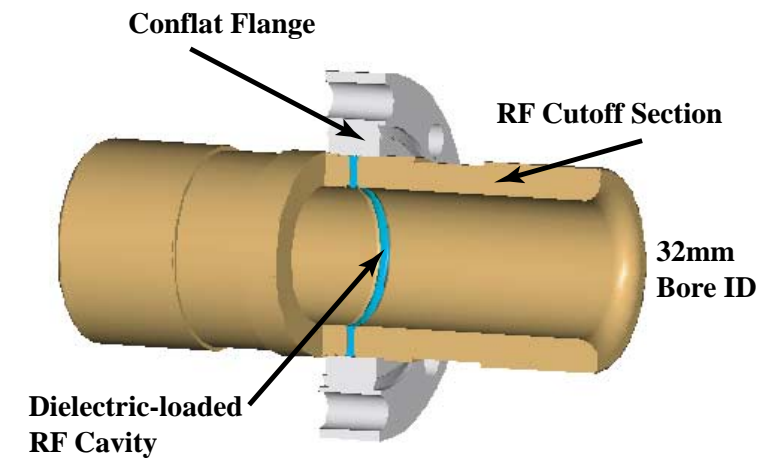

Figure 2: Schematic of dipole-mode pillbox cavity.

\subsection{Capacitive Button Beam Position Monitors}

The capacitive button BPMs copy the same basic construction as the RF cavities. They are built from single conflat flanges, with a brazed stainless steel cutoff section that acts as a high-pass filter. The BPMs themselves are standard button-type. The BPM button electrode is made of 0.125 " OD OFHC copper stock, and inserted within a 0.3 " ID bore hole. This bore hole penetrate from the outer surface of the flange through the inner surface of the brazed-on stainless steel pipe insert. A $50-\Omega$ coaxial SMA vacuum feedthrough is welded onto the outer surface of the flange. From there, individual button signals are collected and either sent directly to the data acquisition oscilloscopes, or are combined with a set of $2-4 \mathrm{GHz}$, $180^{\circ} 3-\mathrm{dB}$ microwave hybrids (Anaren 30056) which then transmit $\Delta \mathrm{x}, \Delta \mathrm{y}$, and sum signals after rectification with microwave detectors (Agilent $8474 \mathrm{C}$ ). A schematic of the BPM is shown in Figure 3. The $-3 \mathrm{~dB}$ knee in the button response transfer function is located at $\sim 1.2 \mathrm{GHz}$. With a $50-\mathrm{W}$ load and $2.6 \mathrm{pF}$ button shunt capacitance, the RC time constant is $\sim 0.13 \mathrm{~ns}$.

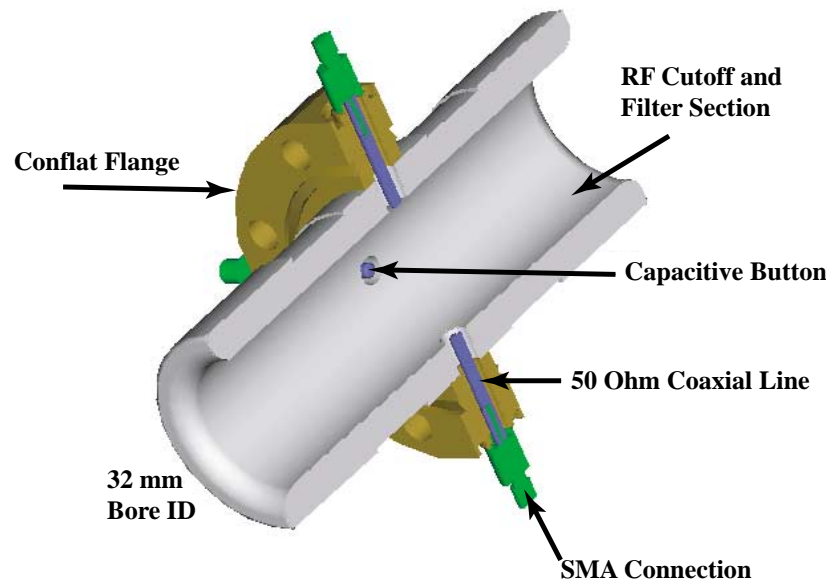

Figure 3: Schematic of capacitive BPM.

\section{MEASUREMENTS}

\subsection{Cavity Mode Parameters}

The electromagnetic simulation and design code GdfidL [2] was used to design the RF cavities. For a $2 \mathrm{~mm}$ long gap, and with dielectric loading, GdfidL predicts a dipole, $\mathrm{TM}_{110}$ mode with frequency $\sim 3.75 \mathrm{GHz}$. The normalized, transverse shunt-impedance, $\left[\mathrm{Z}_{\mathrm{t}} / \mathrm{Q}\right]=[\mathrm{R} / \mathrm{Q}] /\left(\mathrm{k}^{2} \mathrm{x}^{2}\right)$, is then given to be $\sim 13 \Omega$.

A microwave vector network analyzer (HP 8510) was used to measure the dipole mode frequency and Q-values. A $100-\Omega$, two-wire transmission line was used to measure the monopole and dipole scattering parameters from 2-4 $\mathrm{GHz}$. From the $\mathrm{S}_{12}$ parameters were measured the cavity frequencies and Q-values. The measured values of the 10 cavities used in the beamline are listed in Table 1 .

Table 1: Pillbox Cavity Dipole Mode Parameters

\begin{tabular}{ccc}
\hline Cavity & Frequency $(\mathrm{GHz})$ & Q \\
\hline 1 & 3.76 & 100 \\
2 & 3.794 & 120 \\
3 & 3.754 & 110 \\
4 & 3.925 & 140 \\
5 & 3.85 & 110 \\
6 & 3.99 & 120 \\
7 & 3.715 & 130 \\
8 & 3.377 & 150 \\
9 & 3.5 & 100 \\
\hline
\end{tabular}

The unexpected spread in dipole mode frequency between the different cavities is thought to originate from poor quality control over the grade of Alumina used for the dielectric rings. New rings are currently being manufactured and will soon replace the suspect rings within the cavities.

\subsection{BPM Response Function}

The transfer impedance of the individual BPM buttons is measured using the same microwave test stand as the cavities. The individual responses are combined in 
software to calculate the overall BPM response to transverse beam oscillation in the $2-4 \mathrm{GHz}$ range. The response function is shown in Figure 4.

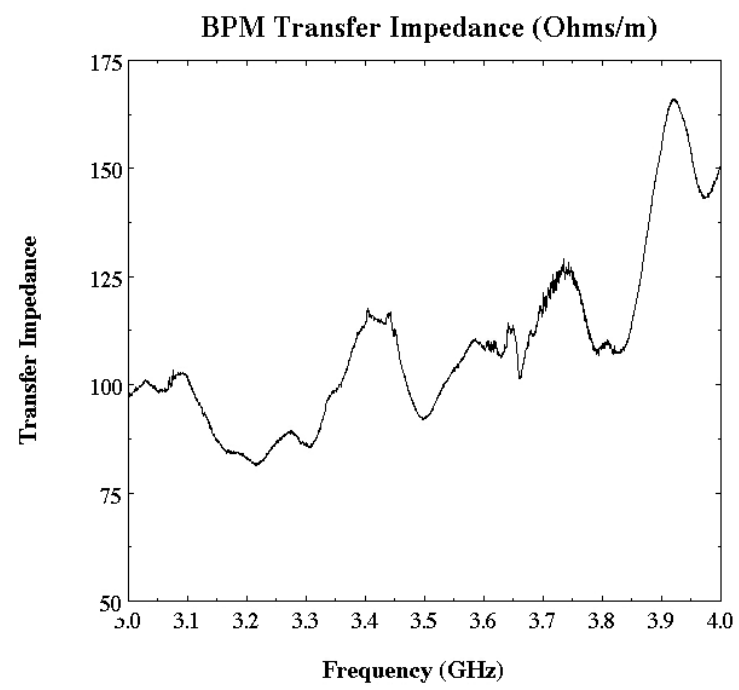

Figure 4: Response function of BPM.

\section{DATA ACQUISITION AND OPERATIONAL BEHAVIOR}

Two different modes of data acquisition were employed during beam operations. The first looked at the rectified signals integrated over the $2-4 \mathrm{GHz}$ microwave hybrid bandpass spectrum. The second looked at the raw BPM signal, mixed down in frequency with a local oscillator (HP 8690B) and monitored with a frequency counter (HP 5351B), then captured on a fast oscilloscope (Tektronix TDS654).

In the first mode, no definite signals were observed in the $2-4 \mathrm{GHz}$ range. This is thought to be due to the small amount of gain of the instability from the lack of overlap between the dipole modes of the different cavities. This mode will be employed again once the dielectrics are replaced in the cavities.

The second mode of measurement displayed a strong signal at approximately $5.35 \mathrm{GHz}$. As shown in reference [1], the transverse dipole instability grows rapidly at this frequency as measured from BPM to BPM along the beamline. Tuning the solenoid transport to the betatron node condition slowed the growth of the instability by more than 13 e-folds over the beamline length.

We are currently measuring a full period mock-up of the BNS beamline on the microwave test stand. GdfidL simulations show a trapped dipole mode in the beam pipe region between RF cavity and BPM that includes the pumping port. Simulations of the empty structure show a dipole mode at $\sim 5.24 \mathrm{GHz}$, and a dipole mode with the 2wire transmission line at $\sim 5.30 \mathrm{GHz}$.

\section{CONCLUSIONS}

We are currently measuring the effectiveness of the Betatron Node Scheme on a simple demonstration beamline. We have presented the design and characterization of the RF components employed in this demonstration. While the trapped dipole mode in the cavity does not seem to produce gain in the BBU instability, we have measured a high frequency trapped mode in the beam pipe. This trapped dipole mode reproduces the BBU instability. Since the trapped modes are identical and occur periodically along the beamline, the growth in the BBU instability can be controlled precisely as if it had originated with the small gap RF cavities themselves.

\section{ACKNOWLEDGMENTS}

We would like to acknowledge the efforts of our chief technicians Wayne Greenway, Eduardo Romero, and David Lozano for their hard work in producing the hardware used in the demonstration experiment. We also appreciate the support of William Barletta, Kem Robinson, Swapan Chattopadhyay, and John Corlett during the design, construction, and operation phases of the experiment. Finally, we thank the members of technical steering committee for their continued support and advice.

\section{REFERENCES}

$\dagger$ SMLidiallbl.gov

[1] T.Houck, S.Lidia, "Beam Dynamics Experiments in Support of Relativistic Klystron", Presented at the 2001 Particle Accelerator Conference, Chicago (2001).

[2] Information on GdfidL can be found at http://www.fbdb.de 